\title{
User-Centric Performance and Cost Analysis for Selecting Access Networks in Heterogeneous Overlay Systems
}

\author{
Bed P. Kafle ${ }^{1}$, Eiji Kamioka ${ }^{1,2}$, and Shigeki Yamada ${ }^{1,2}$ \\ 1 Department of Informatics, The Graduate University for Advanced Studies, \\ 2-1-2 Hitotsubashi, Chiyoda-ku, Tokyo 101-8340, Japan \\ kafle@grad.nii.ac.jp \\ 2 National Institute of Informatics, \\ 2-1-2 Hitotsubashi, Chiyoda-ku, Tokyo 101-8340, Japan
}

\begin{abstract}
In recent years, a wide variety of wireless access networks that support multimedia services have been emerging with different characteristics. The service areas of many of these networks overlap so that a mobile user from an overlapped service area can access any network that supports the user's application. A mobile user can take advantage of the availability of such heterogeneous multimedia networks only when the user terminal is equipped with a mechanism that can select an optimal network for the application. This paper proposes a novel analytical framework of such a mechanism. For this purpose we define the user-centric performance and user-centric cost, and derive their expressions. The user-centric performance, which is also a measure of the user-perceived quality of service (QoS), relates the requirements of user applications with the parameters of network services. The user-centric cost is measured by the user's willingness to pay the price and allow for the consumption of the mobile terminal's resources for accessing the network services. Based on the performance and cost analyses, we describe a network selection mechanism and discuss its implementation issues. We then present an example system to numerically elaborate the functioning of the proposed mechanism.
\end{abstract}

\section{Introduction}

The next generation of mobile communication networks is expected to be a heterogeneous multimedia system widely comprising different radio access networks. Each access network may possess some advantages over the others in terms of network characteristics, such as bandwidth, coverage, cost, and reliability. To exploit these advantages, the heterogeneous system appears in an overlay form [12]; one access network (e.g. wireless LAN) overlapping the service area of the other access networks (e.g. $3 \mathrm{G}$ networks). In such an environment, a mobile host with multi-mode network interfaces should be capable of carrying out the following two functions: (1) selecting an optimal access network and (2) transferring connections from one access network to others when the previous one becomes 
sub-optimal or unavailable. This paper focuses on the first function, i.e. the selection of an optimal network. For this purpose, we (a) define user-centric performance, (b) define user-centric cost, and (c) present a mechanism for selecting an optimal network based on a performance-cost analysis.

There has been a lot of research on the evaluation of network-centric performance or network-level quality of service (QoS), which is concerned with optimizing the network characteristics. The network-centric evaluation indicates, for instance, that the larger the bandwidth, the better the network performs. However, it cannot answer the following question: how much large bandwidth and smaller latency or loss rate are appropriate for a user's application. To answer this question, we need to evaluate the user-centric performance. The user-centric performance, which is also a measure of the user-perceived QoS, relates user application requirements with the network service characteristics or quality. Note that a user requires network resources to be just sufficient enough to satisfy its application's requirements. Any extra resource beyond the requirement, may not give any additional benefit to the user. In such cases, users may not opt for a network that has the highest resources; they may rather select a network that provides the optimal performance at the lowest user-centric cost. The user-centric cost includes the price of network service as well as the resource consumption, such as the battery power of the mobile terminal. It is indisputable that a mechanism should exist that enables mobile users to carry out intelligent decisions for optimal network selection. Without such a mechanism, mobile users cannot get the benefits from the availability of different types of networks; instead they would be overloaded with choices.

There are only a few published research papers dealing with this issue from different aspects. Lee et al. 3] applied a software agent based approach in a personal router (PR) that lies between the user and network. The PR selects a suitable service for the user based on the network information and user preferences. The PR continuously gets feedback of the user's subjective evaluation in terms of quality and cost of service, and accordingly adjusts the user preference parameters. However, their paper does not provide any quantitative analysis of the relationship between the user's requirements and the network's information. Moreover, the subjective evaluation of a particular user cannot be universally valid to all users. Altmann et al. 44 explained a mechanism for enabling users to select a service from a fixed number of priority-level/price pairs provided by a single network. Their paper is more concerned with improving the efficiency of switching service levels when the observed network performance changes than evaluating the user-centric performances.

Complementing the above work, we develop and evaluate an analytical framework for selecting an optimal network in a heterogeneous wireless network environment. For this purpose, we define the user-centric performances and usercentric costs and derive their expressions. The user-centric performance is obtained by relating the network attributes with the requirements of the user applications. We take into consideration three types of attributes - bandwidth, latency, and delay - and derive the user-centric performance for three classes 
of applications - rigid, adaptive, and elastic. Similarly, we consider the price of the network service as well as the power consumption of the mobile terminal to estimate the user-centric cost. Based on the performance and cost analyses, we present a network selection mechanism and discuss its implementation issues.

This paper is organized as follows. In Section 2 we analyze the user-centric performances and user-centric costs. The implementation issues of the proposed network selection mechanism are described in Section 3. We present an example of the performance of the network selection mechanism in Section 4, and a summary and plan for future work in Section 5 .

\section{Performance and Cost Analyses}

\subsection{User-Centric Performance}

We define the user-centric performance (UcPerf) as the degree of fulfillment of user requirements by the network characteristics. There are the following two issues associated with UcPerf analysis.

- The relationship between the UcPerf and network characteristics: how the UcPerf can be expressed as a function of the network characteristics. For instance, how the UcPerf increases as the bandwidth increases or delay decreases.

- The combination of the UcPerf of the characteristics to get an overall UcPerf how to combine the performance of each characteristic to get an overall UcPerf.

As a first step towards these issues, we define a UcPerf as a continuous function of network characteristics because the continuous functions are easier to generate and manipulate than other types of functions. We take the weighted sum of the UcPerf of the characteristics to get an overall UcPerf, because the weights enable us to control the contribution of the individual characteristic on the overall performance. Suppose that there are N wireless networks, each having some different characteristics from the others. Let $\varphi_{x}^{a}\left(x_{k}\right)$ be the UcPerf component of characteristic $x$ (with value $x_{k}$ ) for an application $a$ in a network $k(k \in N)$. Then the overall UcPerf of the network, $U c P e r f_{k}^{a}$, is given as:

$$
U c P e r f_{k}^{a}=\sum_{F} w_{x} \varphi_{x}^{a}\left(x_{k}\right)
$$

where $w_{x}$ is the weighing factor for characteristic $x$ and $F$ is the set of network characteristics that are considered for evaluating the overall UcPerf.

We define $\varphi_{x}^{a}\left(x_{k}\right)$ as a continuous function whose value ranges between 0 and 1; 0 indicating the worst performance and 1 indicating the best performance. The value of the weighing factor $w_{x}$ determines the contribution of the network characteristic $x$ on the overall UcPerf. Considering $\sum_{F} w_{x}=1$ guarantees $0 \leq$ $U c P e r f_{k}^{a} \leq 1$. The shape (or nature) of the $\varphi_{x}^{a}\left(x_{k}\right)$ function depends on both the application type and network characteristic under consideration. We take 
into consideration three types of applications: rigid, adaptive, and elastic. These applications correspond to the following service classes, respectively, defined in the $3 \mathrm{GPP}$ specifications [5].

1. Real-time conversational services: e.g. voice, video telephone, and video gaming, which require minimum bandwidths that must be met, very low delays and no losses - the loss recovery mechanism cannot be effective.

2. Real-time streaming services: e.g. multimedia, video on demand, and webcast, which require adaptive bandwidths, bounded delays and minimum losses.

3. Non real-time interactive and background services: e.g. web browsing, network gaming, database access, email, SMS, and downloading, which require flexible bandwidths, and tolerate delays and losses - the loss recovery mechanism can be used.

Similarly, although UcPerf depends on many networks characteristics, such as bandwidth, latency, loss rate, reliability, availability, coverage, and service provider's reputation, for simplicity, we take into consideration only the first three characteristics, i.e. bandwidth, delay, and loss rate, because these are the most commonly used network characteristics to assess network quality. Let $\varphi_{b}^{a}\left(b_{k}\right), \varphi_{l}^{a}\left(l_{k}\right)$, and $\varphi_{r}^{a}\left(r_{k}\right)$ be the respective UcPerf components derived from bandwidth $\left(b_{k}\right)$, latency $\left(l_{k}\right)$, and loss rate $\left(r_{k}\right)$ in a network $k$ for an application $a$, where $a \in\{$ rigid, elastic, adaptive $\}$. To define $\varphi_{b}^{a}\left(b_{k}\right)$, we take the bandwidth utility functions defined by Shenker [6], and Kafle et al. 7]. In addition, we provide novel definitions of $\varphi_{l}^{a}\left(l_{k}\right)$ and $\varphi_{r}^{a}\left(r_{k}\right)$ based on the specifications of applications as mentioned above.

Rigid application: The UcPerf curves of a rigid application are shown i Fig. 1(a). There can be different sets of functions that can generate these curves. One set of such functions is shown by Equations (2)-(4). As a rigid application requires a minimum amount of bandwidth (say $B_{\min }$ ) to support it, its performance is zero when bandwidth is less than $B_{\min }$. Similarly, it can tolerate a very low network latency of up to $L_{\max }^{\text {rigid }}$ without affecting performance. However, when the delay increases beyond $L_{\max }^{\text {rigid }}$, the performance exponentially degrades at a rate of $\delta_{l}^{\text {rigid }}$. Since these applications are loss intolerant, the performance exponentially decreases at a rate of $\delta_{r}^{\text {rigid }}$ as the loss increases.

$$
\begin{aligned}
& \varphi_{b}^{\text {rigid }}\left(b_{k}\right)= \begin{cases}0 & \text { for } b_{k} \leq B_{\text {min }} \\
1 & \text { otherwise. }\end{cases} \\
& \varphi_{l}^{\text {rigid }}\left(l_{k}\right)= \begin{cases}1 & \text { for } l_{k} \leq L_{\text {max }}^{\text {rigid }} \\
e^{-\left(l_{k}-L_{m a x}^{\text {rigid }}\right) \delta_{l}^{\text {rigid }}} & \text { otherwise. }\end{cases} \\
& \varphi_{r}^{\text {rigid }}\left(r_{k}\right)=e^{-r_{k} \delta_{r}^{\text {rigid }}}
\end{aligned}
$$

Adaptive application: The UcPerf curves of an adaptive application are shown in Fig. 1(b), and the corresponding functions are given by Equations (5)-(7). Adaptive applications adapt their data rate to the available bandwidth in the 

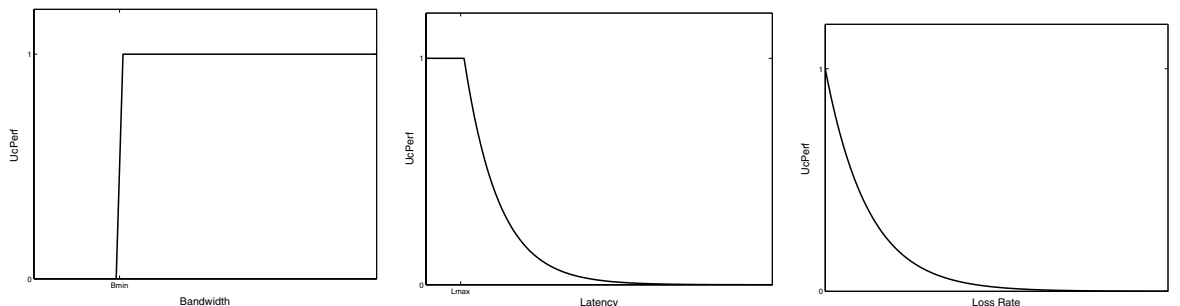

(a) Rigid applications
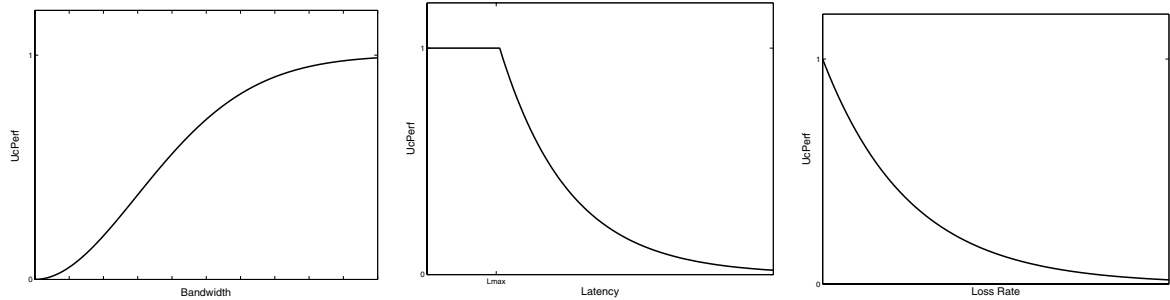

(b) Adaptive applications
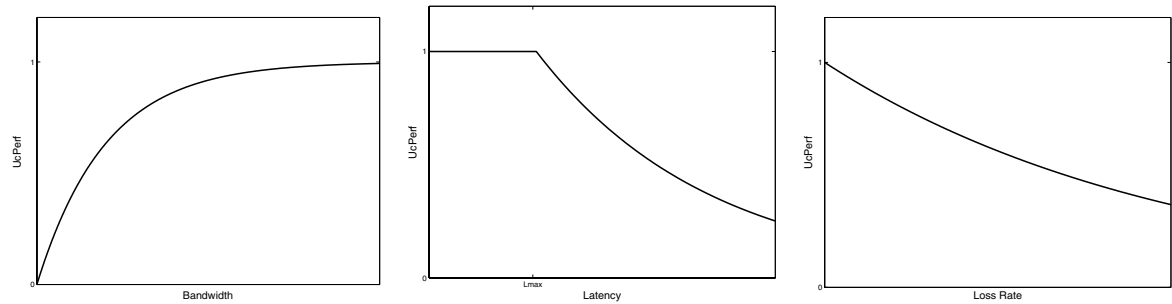

(c) Elastic applications

Fig. 1. User-centric performances $(U c P e r f)$ of bandwidth, latency and loss rate of (a) rigid, (b) adaptive and (c) elastic applications

network and can tolerate occasional delay bound violations and packet losses. However, they have intrinsic bandwidth requirements, as they must maintain the data rate at some minimum level, below which performance suffers badly. This is accounted by a constant $C_{b}$ in Eq. (5); the larger the value of $C_{b}$, the larger the bandwidth required for better performance. Similar to the rigid applications, the latency and loss rate performances of adaptive applications exponentially decrease at the rate of $\delta_{l}^{\text {adaptive }}$ and $\delta_{r}^{\text {adaptive }}$, respectively.

$$
\begin{aligned}
\varphi_{b}^{\text {adaptive }}\left(b_{k}\right) & =e^{-\frac{b_{k}^{2}}{b_{k}+C_{b}}} \\
\varphi_{l}^{\text {adaptive }}\left(l_{k}\right) & = \begin{cases}1 & \text { for } l_{k} \leq L_{\text {max }}^{\text {adaptive }} \\
e^{-\left(l_{k}-L_{\text {max }}^{\text {adaptive }}\right) \delta_{l}^{\text {adaptive }}} & \text { otherwise. }\end{cases} \\
\varphi_{r}^{\text {adaptive }}\left(r_{k}\right) & =e^{-r_{k} \delta_{r}^{\text {adaptive }}}
\end{aligned}
$$

Elastic application: The UcPerf curves of an elastic application are shown in Fig. 1(c), and the corresponding functions are given by Equations (8)-(10). Elastic applications follow the diminishing marginal rate of performance 
improvement as bandwidth increases [6]. This means, when the bandwidth is low, an increment in the bandwidth increases the performance higher than the same increment does when the bandwidth is high. In Eq. (8) $\delta_{b}^{\text {elastic }}$ is the performance increment rate, and $B_{\max }$ is the maximum bandwidth the application can utilize to improve its performance. Similarly, the performances of the latency and loss rate exponentially decrease at the rate of $\delta_{l}^{\text {elastic }}$ and $\delta_{r}^{\text {elastic }}$, respectively.

$$
\begin{aligned}
\varphi_{b}^{\text {elastic }}\left(b_{k}\right) & =e^{-\frac{\delta_{b}^{\text {elastic }} b_{k}}{B_{\text {max }}}} \\
\varphi_{l}^{\text {elastic }}\left(l_{k}\right) & = \begin{cases}1 & \text { for } l_{k} \leq L_{\text {max }}^{\text {elastic }} \\
e^{-\left(l_{k}-L_{\text {max }}^{\text {elastic }}\right) \delta_{l}^{\text {elastic }}} & \text { otherwise. }\end{cases} \\
\varphi_{r}^{\text {elastic }}\left(r_{k}\right) & =e^{-r_{k} \delta_{r}^{\text {elastic }}}
\end{aligned}
$$

Note that since the delay and loss tolerant capacities increase from the rigid to elastic applications, the following relations hold: $L_{\max }^{\text {rigid }} \leq L_{\max }^{\text {adaptive }} \leq L_{\max }^{\text {elastic }}$, $\delta_{l}^{\text {rigid }} \geq \delta_{l}^{\text {adaptive }} \geq \delta_{l}^{\text {elastic }}$, and $\delta_{r}^{\text {rigid }} \geq \delta_{r}^{\text {adaptive }} \geq \delta_{r}^{\text {elastic }}$.

\subsection{User-Centric Cost}

We now derive the expressions of the user-centric costs (UcCost). We take into consideration two types of costs that a user can save: monetary cost and resource cost. The monetary cost includes the price that users have to pay, and the resource cost includes the resources of the user terminal that have to be used for accessing the network services. From the users' point of view, the battery power of a mobile terminal may be a precious resource that the users want to save. For instance, users with lower battery power prefer $3 \mathrm{G}$ networks to wireless LANs as the former consume less power. Similar to the UcPerf, the UcCost is a normalized cost whose value ranges from 0 to 1 ; a 0 indicates that the service cost is trivial, and an 1 indicates that the service cost is the highest of what users are willing to pay.

Let $\vartheta_{p}^{k}\left(p_{k}\right)$ and $\vartheta_{e}^{k}\left(e_{k}\right)$ be the UcCost components of the network service price and battery power (energy) consumption, respectively, in a network $k$ for an application $a$. Then the overall UcCost of the network, $U c C_{o s t}^{a}$, is given as 1

$$
U c \operatorname{Cost}_{k}^{a}=w_{p} \vartheta_{p}^{a}\left(p_{k}\right)+w_{e} \vartheta_{e}^{a}\left(e_{k}\right),
$$

where $w_{p}$ and $w_{e}$ are the weighing factors, such that $\left(w_{p}+w_{e}\right)=1$.

To derive the $U c$ Cost, we use the well-known principle of the demand function of economics. The theory of economics states that the quantity of goods/services demanded (q) increases as the price (p) decreases [8]. As shown by the empirical results in 910, the demand function of a communication service is:

$$
q=A p^{-E},
$$

\footnotetext{
${ }^{1}$ Although we have assumed only two costs: network price and battery power consumption, our model can be extended to more costs cases by introducing additional weights and cost functions.
} 
where $A$ is the scaling constant that is equal to the value of $\mathrm{q}$ when $\mathrm{p}=1$, and $E$ is the constant elasticity of demand for the given service. $E$ is defined as the negative ratio of the relative change in demand to the relative change in price, that is:

$$
E=-\frac{\Delta q / q}{\Delta p / p},
$$

where $\Delta q$ is the change in demand and $\Delta p$ is the change in price. Since the demand increases (decreases) as prices decrease (increase), $\Delta q$ and $\Delta p$ have opposite signs. Therefore, the value of $\mathrm{E}$ is always positive. As estimated by France Telecom, the elasticity, E, for a voice service is 1.337 [10].

An important property of the constant elasticity in Equation (12) is that it creates a demand curve that has different slopes in different price regions. When the price is lower, small changes in price create larger changes in the quantity demanded. On the other hand, when the price is higher, even a large change in price creates only a small change in demand. The demand function can also be interpreted in terms of a utility function. A utility function measures the willingness of users to pay an amount of money for a service with a certain performance or QoS guarantee [11. When the price is lower, users think that the utility of the service is higher than the price paid, so they demand more quantity. On the other hand, when the price is higher, the utility of the service becomes smaller than the price paid, so that users demand less quantity. Based on this assessment, we can express the utility function $(u)$ of the service in terms of the amount of quantity demanded as: $u=q$ when $A=1$, so that $0 \leq u \leq 1$. A high utility indicates that the user is more satisfied, hence the user-centric cost is low. Similarly, a low utility indicates that the user is less satisfied, hence the user-centric cost is high. Therefore, the user-centric cost of the price can simply be expressed as inversely proportional to the service utility. That is,

$$
\vartheta_{p}^{a}(p)=\frac{C}{u}
$$

where $C$ is the proportionality constant. To keep the value of $\vartheta_{p}^{a}(p)$ within the range of 0 to 1 , we take $C=q_{\min }$, where $q_{\min }$ is the minimum amount of service demanded when the price is maximized $\left(p_{\max }\right)$. When we plot the utility and $\vartheta_{p}^{a}(p)$ on a y-axis, and the price on an x-axis, we get the curves shown in Fig. 2 ,

Now we define $\vartheta_{e}^{a}(e)$ as a function of the battery power consumption $(e)$ of the mobile terminal. As there are no references available on how users behave for different levels of power consumption, for the sake of simplicity, we assume that $\vartheta_{e}^{a}(e)$ varies with the battery power consumption in the same way as $\vartheta_{p}^{a}(p)$ does with the price. This means, we suppose that the elasticity of power consumption is the same as that of the network service price.

We use UcPerf and UcCost to estimate the performance-cost ratio (PCR) as given by Eq. (15). The PCR is used as the decision metric to select an optimal access network (that has the maximum value of PCR).

$$
P C R_{k}^{a}=U c P e r f_{k}^{a} / U c \operatorname{Cost}_{k}^{a} \quad \text { for } k=1,2,3, \ldots, N,
$$




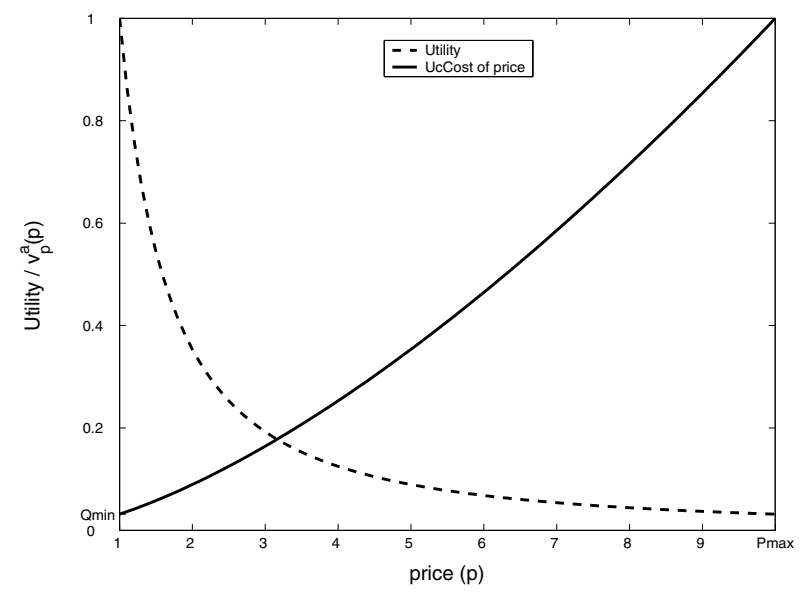

Fig. 2. Utility and UcCost of price $\left(\vartheta_{p}^{a}(p)\right)$ versus network price

where $N$ is the number of access networks available from the location of the mobile user.

\section{Implementation Issues}

The optimal network selection mechanism can be implemented as a module that comprises a number of profiles (Fig. 3). These profiles are described below.

Network profile: The network profile includes the values of network attributes, e.g. bandwidth, latency, loss rate, and prices. The mobile terminal can obtain these values either by monitoring all the available networks in its surrounding area or by consulting a single entity or network that can provide the attributes of all the networks, depending on the architecture of the heterogeneous system. The MIRAI architecture 2] suggests that in heterogeneous system, it is possible to have a basic access network, separate from other wireless networks, for using as a means for wireless access discovery, signaling, and other network management functions. If such a basic access network exists, it can be consulted to get the network characteristics of all access networks in the vicinity of the mobile user.

Application profile: The application profile maintains the values of parameters, such as $B_{\min }, L_{\max }, \delta_{r}$, and $\delta_{l}$, related to the application requirements. These parameters vary from application to application, even within the same type of applications. For instance, the minimum bandwidth requirement of a rigid application depends on whether the application is voice phone, videophone, or video gaming. These parameters of an application can be defined while the application is designed or developed, by observing the effect of the parameter changes on the application quality. These parameters are provided to the application profile when the application is installed in the mobile terminal. Alternatively, these parameter values can be supplied by an independent network 


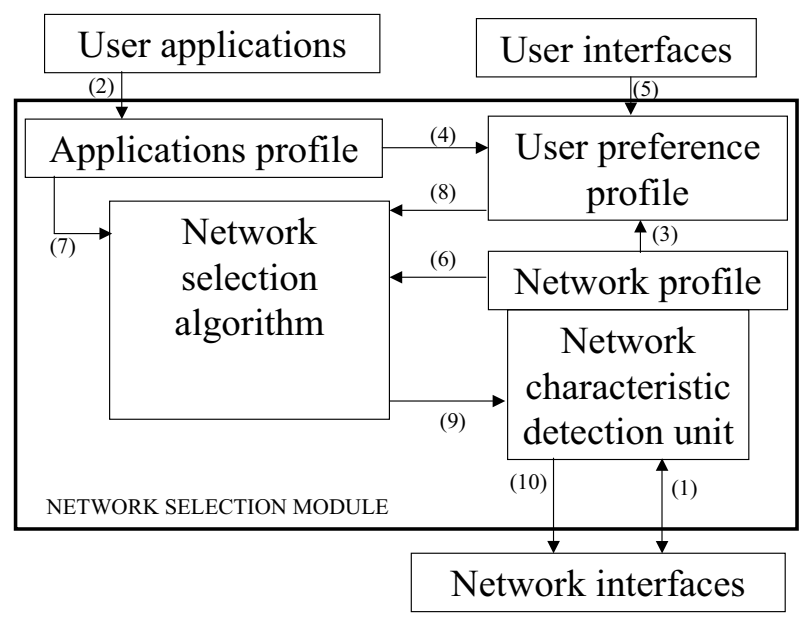

Fig. 3. Interactions among the components of the network selection module

entity similar to the DNS server of current Internet system providing the domain name to IP address mapping information.

User preference profile: The user preference profile maintains the values of the weighting factors used in the evaluations of the user-centric performance and cost. These factors give the notion of the relative importance of the components of $U c P e r f$ and $U c C$ ost. For instance, if a user considers the network price is more important than the battery power consumption, it sets the values of $w_{p}$ and $w_{w}$ in such a way that $w_{p}>w_{w}$. The update of a user preference profile can be done by the users themselves through user interfaces or done automatically (by mobile terminals) by interacting with the application profile, network profile, and user and device contexts.

The components of the network selection module are shown in Fig. 3. In addition to the different profiles, the module includes the network selection algorithm and network characteristic detection unit. In this figure, arrows with numbers in parentheses show the interaction between the different components of the module. We describe these interactions one by one, starting from (1). The network characteristics detection unit probes the available networks in its surrounding area through network interfaces and stores the collected attributes of the networks in the network profile. The application profile interacts with the user applications to collect the application related parameters. The user preference profile consults the network profile, application profile, and user interfaces to maintain the up-to-date values of the user preference parameters based on the supported applications and available networks. The network selection algorithm gets the required information from the network profile, application profile, and user preference profile to compute the UcPerf and UcCost, which are used as the decision metrics to select the best access network. After determining the best access network for the given application, the network selection algorithm request 
the network characteristic detection unit to activate the relevant network interface card. As this module interacts with the user interfaces and applications as well as network interfaces, it can efficiently be located as a middleware between the application and transport layers. We will implement the proposed mechanism in an experimental system and evaluate the performance to validate the theoretical model in our future work.

\section{Numerical Example}

To evaluate the proposed network selection mechanism, we present an example system that has network, application, and user preference profiles as shown in Tables 1, 2, and 3, respectively. We took into consideration two wireless networks, $\mathrm{N} 1$ and N2, and two applications, rigid - voice and adaptive - webcast.

Table 1 shows the network profile containing the attribute values of the network characteristics. Network N1, such as a GSM network, has a smaller bandwidth support ( $b=10 \mathrm{kbps})$ and network $\mathrm{N} 2$, such as a $3 \mathrm{G}$ network, has a higher bandwidth support ( $b=2 \mathrm{Mbps}$ ). Network N2 has a higher bandwidth $(b)$, a lower latency $(l)$, and a lower loss rate $(r)$, but has a higher price $(p)$ and a higher power consumption $(e)$ than network N1. The units of the service price can be in Japanese yen (or US dollors, etc.) per unit of connection time and/or data volume. As we assumed that both price-related and power consumptionrelated components of user-centric cost vary in the same way, the units of power consumption are chosen in such a way that the values of the power consumption and price become comparable. That means the units of price and power consumption are adjusted so that these values are close to each other. For instance, if the network service price is 10 Yen, and power consumption is 100 milliwatts,

Table 1. Network profile: attributes

Table 2. Application profile: parameters

\begin{tabular}{|c|c|c|c|c|c|}
\hline Network $(\mathrm{k})$ & $\mathrm{b}$ & $\mathrm{l}$ & $\mathrm{r}$ & $\mathrm{p}$ & $\mathrm{e}$ \\
\hline $\mathrm{N} 1$ & $14 \mathrm{kbps}$ & $0.12 \mathrm{~s}$ & $0.1 \%$ & 10 & 10 \\
N2 & $2000 \mathrm{kbps}$ & $0.10 \mathrm{~s}$ & $0.1 \%$ & 13 & 15 \\
\hline
\end{tabular}

\begin{tabular}{|c|c|c|c|c|c|}
\hline Application & $B_{\min } / C_{b}$ & $L_{\max }$ & $\delta_{l}$ & $\delta_{r}$ & $\mathrm{E}$ \\
\hline voice & $10 \mathrm{kbps}$ & $0.1 \mathrm{~s}$ & 2.0 & 2.0 & 1.5 \\
webcast & 1000 & $1.0 \mathrm{~s}$ & 1.0 & 1.0 & 1.5 \\
\hline
\end{tabular}

Table 3. User preference profile: parameters

\begin{tabular}{|c|c|c|c|c|c|c|c|}
\hline Application & $w_{b}$ & $w_{l}$ & $w_{r}$ & $w_{p}$ & $w_{e}$ & $p_{\max }$ & $e_{\max }$ \\
\hline voice & 0.6 & 0.3 & 0.3 & 0.8 & 0.2 & 20 & 20 \\
webcast & 0.6 & 0.3 & 0.3 & 0.8 & 0.2 & 20 & 20 \\
\hline
\end{tabular}

Table 4. Decision metrics

\begin{tabular}{|c|c|c|c|c|}
\hline Application & Network & UcPerf & UcCost & PCR \\
\hline voice & N1 & 0.96 & 0.35 & $\mathbf{2 . 7 0}$ \\
voice & $\mathrm{N} 2$ & 0.98 & 0.55 & 1.79 \\
\hline webcast & $\mathrm{N} 1$ & 0.49 & 0.35 & 1.38 \\
webcast & $\mathrm{N} 2$ & 0.99 & 0.55 & $\mathbf{1 . 8 0}$ \\
\hline
\end{tabular}


then the unit of power consumption is converted to a deciwatt so that the value of the power consumption becomes 10 deciwatts.

Table 2 shows the application profile. Here the elasticity $(E)$ of both voice and webcast services are taken as 1.5. The minimum bandwidth $\left(B_{\min }\right)$ required to support the voice service is taken to be $10 \mathrm{kbps}$, and the constant $C_{b}$ of the adaptive webcast application is taken as 1000. Similarly, Table 3 shows the user preference profile. We assumed that user preferences (weighing factors and the maximum amount of price and battery energy that the user can afford) are the same for both applications under consideration.

We evaluated the UcPerf and UcCost of these applications in networks N1 and N2 and listed them in Table 4. It shows that the UcPerfs of the voice application are comparable in both networks, whereas the UcCost is much higher in network N2 than in network N1, because of the higher price and power consumption in network N2. Therefore, the PCR in network N2 smaller than that in network N1. Thus, network N1 is better for the voice application. On the other hand, when we consider an adaptive webcast application, the UcPerf in network N1 is smaller than that in network N2, because the webcast application requires a higher bandwidth for better performance. In this case, the PCR in network N2 is higher than that in network N1, resulting in the selection of network N2 for the webcast application. The UcCosts are the same for both applications, as we have used the same user preference profile.

In our evaluation, not only the network performances, but also the network costs greatly affect the network selection decision. For instance, if the price of the second network (N2) is raised from 13 units to 17 units, keeping other parameters the same, the UcCost in N2 for the webcast application rises to 0.76 (from 0.55) and consequently the PCR falls to 1.31 (from 1.80). This change in price results in the selection of network N1, as its PCR (1.38) is higher than the PCR (1.31) of network N2.

\section{Conclusion}

We developed the theoretical framework of a mechanism that carries out optimal network selection decisions based on the user-centric performance and user-centric cost analyses. The user-centric performance is estimated by comparing the network attributes with the user application requirements. Similarly, we considered the price of the network service and the power consumption of the mobile terminal to estimate the user-centric cost. We presented a network selection mechanism and discussed the relevant issues. Through an example, we numerically illustrated the performance of the proposed mechanism. The limitation of this work is that we have assumed that a user activates only one application at a time and selects the best network for the application. Our model needs an extension to address the issues of selecting an optimal network for multiple applications running simultaneously.

In this work we concentrated on the theoretical work on selecting an optimal network when the user application is initiated. In future work, we will carry 
out a simulation experiment to strengthen the proposed network selection algorithm. We will also evaluate the computational and communicational overheads introduced by the proposed mechanism.

\section{References}

1. E. A. Brewer et al., "A network architecture for heterogeneous mobile computing," IEEE Personal Commun., pp.8-24, Oct. 1998.

2. G. Wu, M. Mizuno, and P. J. Havinga, "MIRAI architecture for heterogeneous networks," IEEE Commun. Mag., pp.126-134, Feb. 2002.

3. G. Lee, P. Faratin, S. Bauer, and J. Wroclawski, "A user-guided cognitive agent for network service selection in pervasive computing environments," IEEE PerCom 2004 .

4. J. Altmann, H. Daanen, H. Oliver, and A. S.-B. Suarez, "How to market-manage a QoS network," IEEE InfoCom, 2002.

5. 3GPP TS 23.107, "Quality of service (QoS) concept and architecture," Version 6.1.0, April 2004.

6. S. Shenker, "Fundamental of design issues for the future Internet," IEEE J. Sel. Areas Commun., vol.13, no.7, pp.1176-1188, Sept. 1995.

7. V. P. Kafle, E. Kamioka, and S. Yamada, "Maximizing user satisfaction based on mobility in heterogeneous mobile multimedia communication networks," IEICE Trans. Commun., vol.E88-B no.7, pp.2709-2717, July 2005.

8. N. G. Markiw, Essential of Economics, Second Edition, South-western, Thomson Learning, 2001, pp.67-98.

9. S. G. Lanning, D. Mitra, Q. Wang and M. H. Wright, "Optimal planning for optical transport networks," Philosoph. Trans. Royal Soc. London A, vol.358, no.1773, pp.2183-2196, Aug. 2000.

10. M. Aldebert, M. Ivaldi, and C. Roucolle, "Telecommunication demand and pricing structure: an econometric analysis," Telecommunication System, Kluwer, vol.25, no.1-2, pp.89-115, 2004.

11. L. A. DaSilva, "Pricing for QoS-enabled networks: a survey," IEEE Commun. Surveys, http://www.comsoc.org/pubs/surveys, Second Quarter 2000. 